commonly due to blood loss from lesions in the gastrointestinal (GI) tract and malabsorption, accounting for $4-13 \%$ of referrals to gastroenterologists. In men over the age of 50 years and postmenopausal women there is established data on the incidence of GI pathologies causing IDA but such data for men under 50 years of age is not as robust. We carried out a retrospective analysis of outcomes of investigations for IDA in men under the age of 50 years over a period of 10 years in our hospital serving about 325,000 population.

Methods Through the audit and clinical code department all male patients between 17 and 50 years of age investigated for IDA from 2000-2010 were identified retrospectively. The criteria used for diagnosis of IDA included haemoglobin level below the lower limit of normal, low ferritin and corresponding abnormalities of red cell indices. Data on outcome of investigations for IDA was collected from patient case notes and endoscopy, radiology and pathology records.

Results 52 patients were identified over the study period. The median haemoglobin was $9.3 \mathrm{~g} / \mathrm{dl}$. The median age of the patients was 44 years. 44/52 (85\%) had investigations recorded. 18/52 (33\%) had gastroscopy (OGD) only. 26/52 (48\%) had both OGD and colonic investigations. 7/52 (13\%) had further investigations following normal bi-directional endoscopy including bone marrow, small bowel barium studies, capsule endoscopy and abdominal ultrasound with none of these additional investigations yielding further diagnostic information. With regards to colonic investigations $21 / 26$ (81\%) had colonoscopy, $3 / 26(11 \%)$ had barium enema plus flexible sigmoidoscopy and $2 / 26(8 \%)$ had CT scan.

The findings of OGD were normal investigation 25/44 (57\%), oesophagitis 5/44 (12\%), peptic ulcer disease (PUD) 4/44 (9\%), hiatus hernia $4 / 44$ (9\%), oesophageal cancer $1 / 44(2 \%)$ and coeliac disease $2 / 25$ patients with duodenal biopsies at OGD.

The findings of lower GI investigations were normal investigations $16 / 29(55 \%)$, haemorrhoids $5 / 29(17 \%)$, inflammatory bowel disease (IBD) $3 / 29(10 \%)$, polyps $2 / 29(7 \%)$, colorectal cancer $2 / 29$ (7\%) and diverticulosis $1 / 29$ (4\%).

Conclusion Significant findings including PUD, malignancy and IBD constituted $19.2 \%$. Malignancy accounted for $5.8 \%$ and this was comparable with previously reported prevalence of GI malignancy in patients with IDA (6-13\%). In addition a proportion of investigations also yielded other diagnosis including oesophagitis and coeliac disease. Therefore it will be justified to investigate men under 50 years with IDA similarly to those over 50 years and postmenopausal women as suggested in most international guidelines

Disclosure of Interest None Declared.

\section{PWE-191 IS GASTROPEXY AN ALTERNATIVE TO RADIOLOGICAL GASTROSTOMY? A SINGLE CENTRE EXPERIENCE}

doi:10.1136/gutjnl-2013-304907.479

1,*S S Salunke, 'D Barber, 'R McKay, 'A W McKinlay, ' IJ S Leeds. 'Gastroenterology, Aberdeen Royal Infirmary, Aberdeen, Aberdeen, UK

Introduction Standard inside-out PEG insertion is not always technically possible or safe especially when there is narrowing of the oesophagus or pharynx with head-and-neck or oesophageal cancers. There is also concern about tumour seeding with inside-out technique. Similarly, in some patients it is not possible to pass the standard gastroscope through to upper GI tract. Gastropexy is an alternative technique which allows insertion of a gastrostomy tube with outside-in technique and can be performed using slimmer scopes. Gastropexy has been routinely performed in our unit for some time and therefore we aimed to review the experience of Gastropexy insertion in our unit.

Methods Gastropexy placement in our unit is based upon a previously described technique using Kimberly Clark MIC introducer kit. A standard endoscopy is performed by the oral or nasal route, a site identified and the stomach secured against the anterior abdominal wall with 3 pre-loaded T-toggles which can be fastened with a locking disc. A tract is formed using a single serial dilator passed over a guidewire and a $14 \mathrm{~F}$ balloon gastrostomy inserted through the dilator and secured. The outer sheath of the dilator will then be peeled out. All patients receive pre-procedural prophylactic antibiotics. A retrospective review of all gastropexy procedures between June 2009 and November 2012 was carried out. Patient demographics, indication, sedation requirements and complication rates were recorded.

Results 45 procedures were carried out on 42 patients (28 males, median age 63 years range $56-84$ ) with a technical success rate of $95.7 \%$ for placement. Indication for placement was head-and-neck cancer $(n=34)$, oesophageal stricture/cancer $(n=9)$ and neurologi$\mathrm{cal}(\mathrm{n}=2) .17 \%$ of procedures were performed under general anaesthesia as part of another surgical procedure with the remainder having conscious sedation (mean doses midazolam $3.8 \mathrm{mg}$ and pethidine $17.8 \mathrm{mg}$ ). $58 \%$ of procedures were performed using a nasal/ neonatal endoscope. Of these, $62 \%$ cases had head-and-neck cancer, $31 \%$ had oesophageal cancer/stricture. One patient had a minor gastric fluid leak and one patient developed a pneumoperitoneum both of which were managed conservatively. At 7 days, 1/45 (2.2\%) had a site infection and 1/45 (2.2\%) had died whereas at 28 days, $5 / 45(11.1 \%)$ had a site infection and $4 / 45$ (8.8\%) had died. Mortality at 1 year was $48 \%$, with median survival of 5 months. The primary pathology in all the patients who died was head and neck or oesophageal cancer. None of the deaths were procedure related.

Conclusion Gastropexy is a suitable alternative in patients with difficult access and can be inserted with high success rate and low complication rates. Ideally, a randomised trial comparing gastropexy and radiological gastrostomy insertion should be undertaken. Disclosure of Interest None Declared.

\section{PWE-192 DOES FIBRE REDUCE THE RISK OF CLOSTRIDIUM DIFFICILE DIARRHOEA IN NASOGASTRIC FED PATIENTS?}

doi:10.1136/gutjnl-2013-304907.480

1,"S Antoniou, 'L Goddard, 'C Pettitt, 'T Orchard, 'I Tyrrell-Price. 'Imperial College Healthcare NHS Trust, London, UK

Introduction Tube-feeding has previously been associated with an increased risk of Clostridium difficile ( $C$. difficile) diarrhoea. ${ }^{1}$ The absence of dietary fibre has been suggested as a possible cause, but not yet formally assessed in patients fed via a nasogastric (NG) tube. The objective was to determine if there is a difference in acquisition of $C$. difficile between fibre and fibre-free NG feeds.

Hypothesis Fibre-free NG feeds are associated with a higher rate of $C$. difficile infection than fibre containing feeds.

Methods This was a Retrospective Cohort Study, using data from NG-fed patients in one trust from May to November 2010.

Results It was found that 8 of 169 patients in the fibre-fed group had C. difficile, compared with 15 of 202 in the non-fibre fed group, equating to $4.7 \%$ and $7.4 \%$ detection of $C$. difficile respectively, $p$ value 0.39 . Antibiotic usage was similar in both groups. Patients who received fibre free feeds were more likely to develop diarrhoea than those receiving fibre containing feeds $(p=0.0112)$.

Conclusion The results show a trend towards fibre reducing the risk of $C$. difficile diarrhoea. However the effect appears to be subtle, as it failed to reach statistical significance despite the inclusion of over 160 patients in both groups. The previous finding of a link between fibre free NG feeding and C. difficile acquisition may have been due to increased rates of diarrhoea. Fibre reduced the risk of diarrhoea, which may reduce the apparent risk of $C$. difficile when compared with the fibre free group, as in the latter there is a potential for $C$. difficile spore shedding from asymptomatic carriers.

Disclosure of Interest None Declared. 